\title{
Validation of Modified ALBI Grade for More Detailed Assessment of Hepatic Function in Hepatocellular Carcinoma Patients: A Multicenter Analysis
}

\author{
Atsushi Hiraoka $^{a}$ Takashi Kumada $^{b}$ Kunihiko Tsujic Koichi Takaguchid \\ Ei Itobayashi ${ }^{\text {Kazuya Kariyama }}{ }^{f}$ Hironori Ochi ${ }^{9}$ Kazuto Tajiri ${ }^{\text {h }}$ \\ Masashi Hirooka ${ }^{i}$ Noritomo Shimadaj Toru Ishikawak Yoshihiko Tachi ${ }^{1}$ \\ Toshifumi Tada $^{b}$ Hidenori Toyodab Kazuhiro Nouso ${ }^{f}$ Kouji Joko $^{g}$ \\ Yoichi Hiasa' Kojiro Michitaka ${ }^{\mathrm{a}}$ Masatoshi Kudom \\ on behalf of the RELPEC Study Group and HCC 48 Group \\ a Gastroenterology Center, Ehime Prefectural Central Hospital, Ehime, Japan; \\ bDepartment of Gastroenterology and Hepatology, Ogaki Municipal Hospital, Gifu, \\ Japan; ' Gastroenterology Center, Teine Keijinkai Hospital, Sapporo, Japan; dDepartment \\ of Hepatology, Kagawa Prefectural Central Hospital, Takamatsu, Japan; e Department of \\ Gastroenterology, Asahi General Hospital, Asahi, Japan; ${ }^{f}$ Department of Gastroenterology, \\ Okayama City Hospital, Okayama, Japan; ${ }^{9}$ Hepato-Biliary Center, Matsuyama Red Cross \\ Hospital, Matsuyama, Japan; h Department of Gastroenterology, Toyama University \\ Hospital, Toyama, Japan; iDepartment of Gastroenterology and Metabology, Ehime \\ University Graduate School of Medicine, Ehime, Japan; jDivision of Gastroenterology and \\ Hepatology, Ootakanomori Hospital, Kashiwa, Japan; kDepartment of Gastroenterology, \\ Saiseikai Niigata Daini Hospital, Niigata, Japan; 'Department of Gastroenterology, Komaki \\ City Hospital, Komaki, Japan; m Department of Gastroenterology and Hepatology, Kindai \\ University School of Medicine, Osaka, Japan
}

\section{Keywords}

Hepatocellular carcinoma - Albumin-bilirubin grade - Modified ALBI - Japan Integrated Staging score $\cdot$ ALBI-T score $\cdot \mathrm{mALBI}-\mathrm{T}$ score $\cdot$ Prognosis

\section{Abstract}

Background/Aim: The frequency of hepatocellular carcinoma (HCC) in patients with good hepatic reserve function has been increasing in Japan along with the progression of antiviral therapies and aging of the society. We evaluated the usefulness of modified albumin-bilirubin (ALBI) grade as a tool for assessment of hepatic reserve function. Materials/Methods: We enrolled 6,649 naïve HCC patients treated from 2000 to 2017 and divided them into training 
(Ehime Prefecture group: E group, $n=2,357$ ) and validation (validation group: $\mathrm{V}$ group, $n=$ 4,292 ) cohorts. Child-Pugh classification and ALBI and modified ALBI (mALBI) grading were compared using with Japan Integrated Staging (JIS), ALBI-TNM (ALBI-T), and mALBI-T scores, which were calculated based on TNM stage and each assessment tool, retrospectively. $\boldsymbol{R e}$ sults: In the E group, Akaike's Information Criterion (AIC) and c-index values for mALBI-T $(13,725.2 / 0.744)$ were better as compared to those of ALBI-T $(13,772.6 / 0.733)$ and JIS score $(13,874.7 / 0.720)$, with similar results observed in the $V$ group (mALBI-T: 27,727.4/0.760; ALBI-T: 27,817.8/0.750; JIS: 27,807.5/0.748). Although there were some significant differences between the groups with regard to clinical background factors (age, etiology, tumor size, tumor number, treatment modalities), for all patients the AIC and c-index values of mALBI-T (45,327.1/0.755) were also better than those of ALBI-T $(45,467.7 / 0.744)$ and JIS scores $(45,555.8 / 0.739)$, indicating its superior stratification ability and prognostic predictive value in patients with $\mathrm{HCC}$. Conclusion: The detailed stratification ability of $\mathrm{mALBI}$ grade for hepatic reserve function is suitable for the recent trend of HCC patients, while mALBI-T may provide a more accurate predictive value than existing total staging scoring systems.

(C) 2018 S. Karger AG, Basel

\section{Introduction}

Hepatocellular carcinoma (HCC) is the second most common cause of cancer-related death worldwide [1]. Recently, eradication of hepatitis C virus (HCV) and control of hepatitis $B$ virus (HBV) with use of direct acting antivirals [2, 3] and nucleotide analogues [4, 5], along with progression in the development of low invasive therapeutic modalities such as ablative therapy (e.g., percutaneous ethanol injection therapy, radiofrequency ablation [RFA] [6-8]) have contributed to the improvement of hepatic reserve function and prognosis in HCC patients. However, an important remaining issue is lack of development of a suitable evaluation tool for hepatic reserve function in this era of hepatitis control. Although Child-Pugh classification [9] has traditionally been used for assessment of hepatic function, its effectiveness has not been established based on a statistical method, as classification is determined using both objective (ascites, hepatic coma) and semiquantitative factors. Previously, the albumin-bilirubin (ALBI) grade obtained with a statistical method was proposed as a new assessment tool [10], and some studies of its usefulness have reported its ability to predict prognosis and assist in decision making regarding treatment choices for HCC patients [1114]. However, ALBI grade 2 includes patients with a wide range of hepatic function as well as those rated as Child-Pugh class B. In a recent report, a modification of ALBI grade (mALBI grade) into 4 subgrades $(1,2 a, 2 b$, and 3$)$ was proposed $[15,16]$. The present validation study aimed to evaluate the usefulness of mALBI grade for predicting the prognosis of HCC patients.

\section{Materials and Methods}

We enrolled 7,898 patients with naïve HCC who were diagnosed and treated from 2000 to 2017 at 12 institutions in Japan (Ehime Prefectural Central Hospital [ $n=1,627]$, Ogaki Municipal Hospital $[n=1,373]$, Teine Keijinkai Hospital [ $n=943]$, Kagawa Prefectural Central Hospital [ $n=894]$, Asahi General Hospital [ $n=811]$, Okayama City Hospital [ $n=400]$, Matsuyama Red Cross Hospital [ $n=376]$, Toyama University Hospital [ $n=368]$, Ehime University Hospital [ $n=365]$, Otakanomori Hospital [ $n=313]$, Saiseikai Niigata Daini Hospital [ $n=284]$, Komaki City Hospital $[n=144]$ ). After exclusion of those with no information regarding tumor node metastasis (TNM) stage, assessed according to the Liver Cancer Study Group of Japan (LCSGJ) criteria 6th edition (Table 1), or Child-Pugh classification, as well as 1 case treated with liver transplantation, we analyzed the clinical characteristics and prognosis of 6,649 patients. Of those, 2,357 were 


\section{Liver Cancer}

Table 1. Tumor node metastasis stage of the Liver Cancer Study Group of Japan

\begin{tabular}{|c|c|}
\hline \multicolumn{2}{|l|}{ Liver Cancer 2019;8:121-129 } \\
\hline DOI: $10.1159 / 000488778$ & $\begin{array}{l}\text { (c) } 2018 \text { S. Karger AG, Basel } \\
\text { www.karger.com/lic }\end{array}$ \\
\hline
\end{tabular}

Hiraoka et al.: Modified ALBI Grade for HCC Treatment
T factor $^{\text {a }}$ for TNM stage of LCSGJ 6th edition

$\mathrm{T} 1$

$\mathrm{T} 2$

T3

$\mathrm{T} 4$

Fulfilling 3 factors

Fulfilling 2 factors

Fulfilling 1 factor

Fulfilling 0 factors
Table 2. JIS, ALBI-T, and mALBI-T scoring systems
TNM stages in LCSGJ 6th edition

Stage I T1NOMO

Stage II T2NOM0

Stage III T3NOMO

Stage IVa T4N0M0, or any TN1M0

Stage IVb Any TN0-N1M1

LCSGJ, Liver Cancer Study Group of Japan; TNM stage, tumor node metastasis stage. ${ }^{\text {a }}$ The 3 factors are a single lesion, a lesion measuring $<2 \mathrm{~cm}$, and no vascular involvement.

\begin{tabular}{lllll}
\hline & Score 0 & Score 1 & Score 2 & Score 3 \\
\hline JIS & & & & \\
$\begin{array}{l}\text { Child-Pugh class } \\
\text { TNM stage }\end{array}$ & A & B & C & \\
\hline$A L B I-T$ & I & II & III & IV \\
ALBI grade & 1 & 2 & & \\
TNM stage & I & II & III & IV \\
\hline $\begin{array}{l}\text { mALBI-T } \\
\text { mALBI grade }\end{array}$ & 1 & & & \\
TNM stage & I & II & III & IV \\
\hline
\end{tabular}

JIS, Japan Integrated Staging; TNM stage, tumor node metastasis according to the criteria of Liver Cancer Study Group of Japan, 6th edition; ALBI-T, albumin-bilirubin grade-TNM; mALBI-T, modified ALBI-TNM.

treated at 3 institutions in the Ehime Prefecture area (Ehime Prefectural Central Hospital, Ehime University Hospital, Matsuyama Red Cross Hospital) and analyzed as the training cohort (Ehime Prefecture group: E group), while the other 4,292 treated at 9 institutions in another area served as the validation cohort (validation group: $V$ group).

\section{Basal Hepatic Disease}

Patients positive for anti-HCV were judged to have HCC due to HCV, and those positive for HBV surface antigen (HBsAg) were judged to have HCC due to HBV. Cases without anti-HCV or HBsAg were defined as negative for both HBV and HCV.

Methods for Assessment of Hepatic Reserve Function and Prognosis

Child-Pugh classification, and ALBI and mALBI grades were used as assessment tools for hepatic reserve function. ALBI grade was calculated based on serum albumin and total-bilirubin values using the following formula: ALBI-score: $\left(\log _{10}\right.$ bilirubin $\left.(\mu \mathrm{mol} / \mathrm{L}) \times 0.66\right)+($ albumin $(\mathrm{g} / \mathrm{L}) \times-0.085)$; and ALBI grade was defined by the score of the following: $\leq-2.60=$ Grade $1,>-2.60$ to $\leq-1.39=$ Grade $2,>-1.39=$ Grade 3 [10]. ALBI grade 2 was further divided into 2 subgrades ( $2 \mathrm{a}$ and $2 \mathrm{~b}$ ) using a previously reported cut-off value (ALBI score -2.270) and the 4 ALBI grades were named as mALBI grade $[15,16]$.

Japan Integrated Staging (JIS), ALBI-TNM (ALBI-T), and mALBI-TNM (mALBI-T) scores were used for evaluation of prognosis, and their predictive ability in regard to prognosis was compared. JIS score was calcu- 
Table 3. Characteristics of all patients

\begin{tabular}{|c|c|c|c|c|}
\hline & Total $(n=6,649)$ & E group $(n=2,357)$ & V group $(n=4,292)$ & $p$ value \\
\hline Age, years & $69.4(9.9)$ & $69.0(10.2)$ & $69.6(9.8)$ & 0.028 \\
\hline Gender, M/F & $4,648 / 2,001$ & $1,675 / 682$ & $2,973 / 1,319$ & 0.116 \\
\hline $\begin{array}{l}\mathrm{HCV} / \mathrm{HBV} / \mathrm{HBV} \& \mathrm{HCV} / \mathrm{Alc} / \\
\text { nonBnonC/UK }\end{array}$ & $4,162 / 818 / 45 / 387 / 1,235 / 2$ & $1,542 / 260 / 22 / 205 / 327 / 1$ & $2,620 / 558 / 23 / 182 / 908 / 1$ & $<0.001$ \\
\hline Child-Pugh class, $\mathrm{A} / \mathrm{B} / \mathrm{C}$ & $4,812 / 1,546 / 291$ & $1,713 / 567 / 77$ & $3,099 / 979 / 214$ & 0.422 \\
\hline ALBI grade, $1 / 2 / 3$ & $2,531 / 3,630 / 488$ & $917 / 1,256 / 184$ & $1,614 / 2,374 / 304$ & 0.563 \\
\hline mALBI grade, $1 / 2 a / 2 b / 3$ & $4,162 / 818 / 45 / 387 / 1,235 / 2$ & $917 / 518 / 738 / 184$ & $1,614 / 934 / 1,440 / 304$ & 0.348 \\
\hline Tumor size, $\mathrm{cm}$ & $3.6(3.1)$ & $3.5(2.9)$ & $3.7(3.2)$ & 0.012 \\
\hline Tumor number, single/multiple & $3,934 / 2,715$ & $1,438 / 919$ & $2,496 / 1,796$ & 0.024 \\
\hline TNM stage, I/II/III/IV & $1,690 / 2,687 / 1416 / 856$ & $601 / 995 / 479 / 282$ & $1,089 / 1,692 / 937 / 574$ & 0.093 \\
\hline JIS score, $0 / 1 / 2 / 3 / 4 / 5$ & $1,337 / 2,364 / 1,581 / 886 / 374 / 107$ & $469 / 873 / 570 / 302 / 114 / 29$ & $868 / 1491 / 1011 / 584 / 260 / 78$ & 0.101 \\
\hline ALBI-T score, $0 / 1 / 2 / 3 / 4 / 5$ & $721 / 2,064 / 1,897 / 1,188 / 624 / 155$ & $260 / 762 / 665 / 400 / 219 / 51$ & $461 / 1,302 / 1232 / 788 / 405 / 104$ & 0.087 \\
\hline mALBI-T score, $0 / 1 / 2 / 3 / 4 / 5 / 6$ & $721 / 1,584 / 1,522 / 1,361 / 812 / 494 / 155$ & $260 / 591 / 537 / 464 / 288 / 166 / 51$ & $461 / 993 / 985 / 897 / 524 / 328 / 104$ & 0.084 \\
\hline Treatment, SR/RFA/PEI/ & & & & \\
\hline TACE/others/BSC & $1,734 / 2,243 / 79 / 1,587 / 423 / 583$ & $563 / 1,075 / 46 / 372 / 73 / 228$ & $1,171 / 1,168 / 33 / 1,215 / 350 / 355$ & $<0.001$ \\
\hline Observation period, years & $3.31(3.11)$ & $3.2(3.01)$ & $3.36(3.17)$ & 0.038 \\
\hline
\end{tabular}

Values for age, tumor size, and observation period are expressed as mean (SD). HCV, hepatitis C virus; HBV, hepatitis B virus; Alc, alcohol; nonBnonC, both negative for HBV and HCV; UK, unknown; ALBI grade, albumin-bilirubin grade; mALBI grade, modified ALBI grade; JIS, Japan Integrated Staging; TNM stage, tumor node metastasis according to the criteria of the Liver Cancer Study Group of Japan, 6th edition; ALBI-T, ALBI-TNM; mALBI-T, mALBI-TNM; SR, surgical resection; RFA, radiofrequency ablation; PEI, percutaneous ethanol injection therapy; TACE, transcatheter arterial chemoembolization; BSC, best supportive care.

lated by summing up scores for Child-Pugh class and TNM stage. ALBI-T and mALBI-T were calculated using either ALBI or mALBI grade and TNM stage, and then calculated in the same manner as JIS (Table 2).

Diagnosis and Treatment of HCC

HCC was diagnosed based on an increasing course of alpha-fetoprotein (AFP), as well as dynamic CT [17], magnetic resonance imaging(MRI) [18, 19], contrast-enhanced US (CEUS) with perflubutane (Sonazoid ${ }^{\circledR}$, Daiichi Sankyo Co., Ltd. Tokyo, Japan) [20, 21], and/or pathological findings. TNM stage was determined as previously reported in studies for staging of HCC conducted by the LCSG] [22] (Table 1). From 2006, all treatments were performed following the Japanese practical guidelines for HCC [23, 24], whenever possible, after obtaining written informed consent from the patient. The present study protocol was approved by the Institutional Ethics Committee of Ehime Prefectural Central Hospital (No. 28-30).

\section{Statistical Analysis}

Data are expressed as the mean and standard deviation (SD). Statistical analyses were performed using Student's $t$ test, a Mann-Whitney U test, a $\chi^{2}$ test, and Kaplan-Meyer methods with a log-rank test. A $p$ value $<0.05$ was considered to indicate statistical significance. For multiple comparisons, Holm's method was used. Akaike's Information Criterion (AIC) and c-index were used for evaluation of ability for stratification and prediction of prognosis by each method. All statistical analyses were performed using Easy R (EZR) (Saitama Medical Center, Jichi Medical University, Saitama, Japan) [25], a graphical user interface for R (The R Foundation for Statistical Computing, Vienna, Austria).

\section{Results}

The characteristics of the present cohort are shown in Table 3. Average age was $69.4 \pm$ 9.9 years, males comprised 69.9\% $(n=4,648)$, and the average observation period was 3.31 \pm 3.11 years (TNM stage I, II, III, IV = 1,690, 2,687, 1,416, 856 cases, respectively; surgical resection, RFA, percutaneous ethanol injection, transcatheter arterial chemoembolization, other, best supportive care $=1,734,2,243,79,1,587,423,583$ cases, respectively).

In the E group, used as the training cohort $(n=2,357)$, the stratification ability regarding prognosis by each system was good (Fig. 1a), though the AIC and c-index values for mALBI-T 


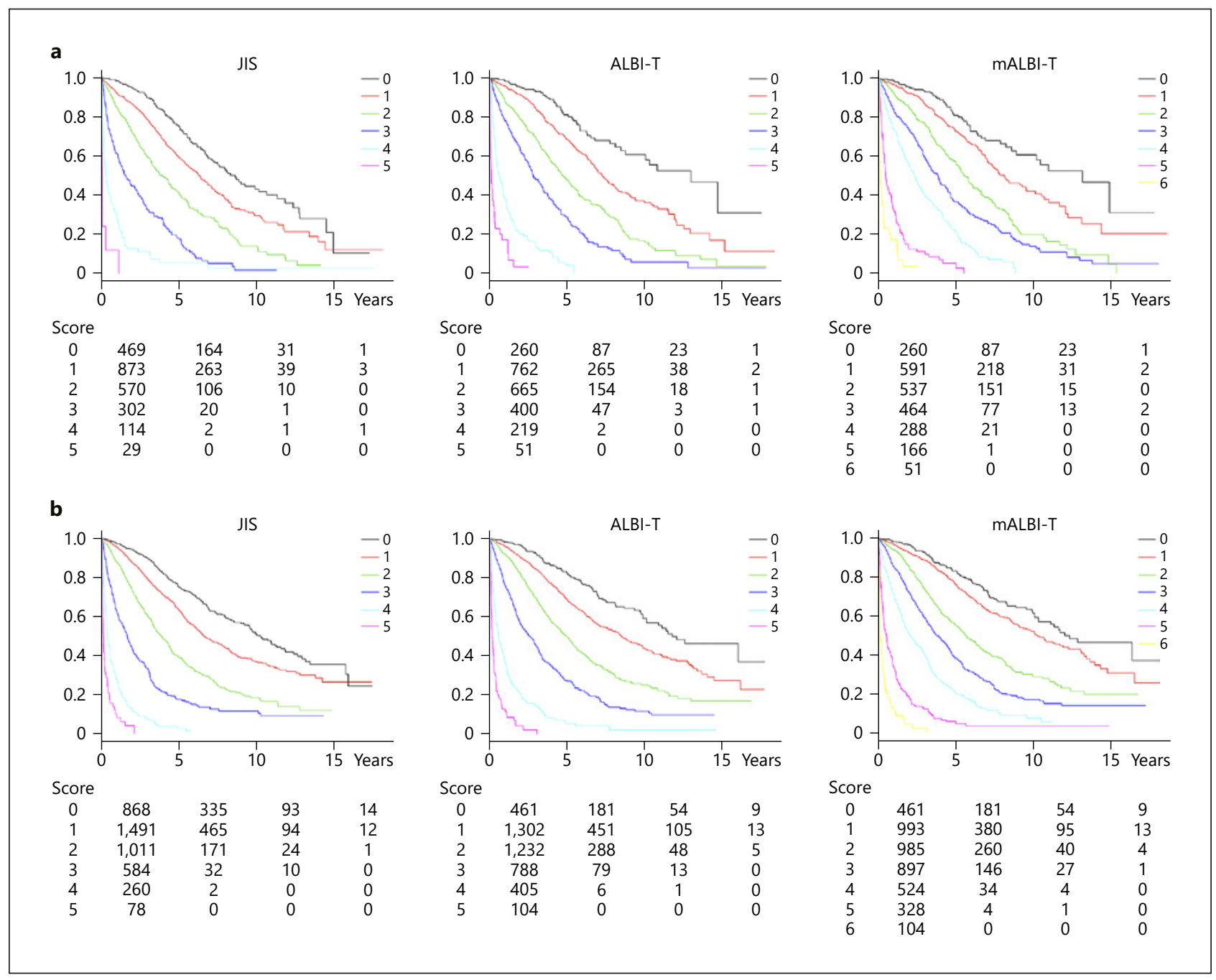

Fig. 1. JIS, ALBI-T, and mALBI-T scores for E and V groups. a The E group was used as a training cohort $(n=$ 2,357). The AIC and c-index values for JIS were 13,874.7 and 0.720, respectively, for ALBI-T were 13,772.6 and 0.733 , respectively, and for mALBI-T were 13,725.2 and 0.744 , respectively. $\mathbf{b}$ The $\mathrm{V}$ group was used as a validation cohort $(n=4,292)$. The AIC and c-index values for JIS were $27,807.5$ and 0.748 , respectively, for ALBI-T were 27,817.8 and 0.750, respectively, and for mALBI-T were 27,727.4 and 0.760, respectively. a, b Values on the $y$ axes represent estimation of cumulative survival probability.

$(13,725.2$ and 0.744 , respectively) were superior to those of ALBI-T $(13,772.6$ and 0.733 , respectively) and JIS $(13,874.7$ and 0.720 , respectively). In the $V$ group, used as the validation cohort $(n=4,292)$, similar results were observed (Fig. 1b), as the AIC values for JIS, ALBI-T, and mALBI-T were 27,807.5, 27,817.8, and 27,727.4, respectively, and their c-index values were $0.748,0.750$, and 0.760 , respectively.

Although there were some significant differences observed regarding some of the clinical background factors between the $\mathrm{E}$ and $\mathrm{V}$ groups (age, etiology, tumor size, tumor number, treatment modalities) (Table 3), mALBI-T showed a better stratification ability and prognostic predictive value with both cohorts, as the AIC and c-index values for mALBI-T were also better than those of the others for all patients (Table 4). The prognosis of patients after dividing by TNM stage could be separated according to the order of mALBI grade $(p<0.001$, 


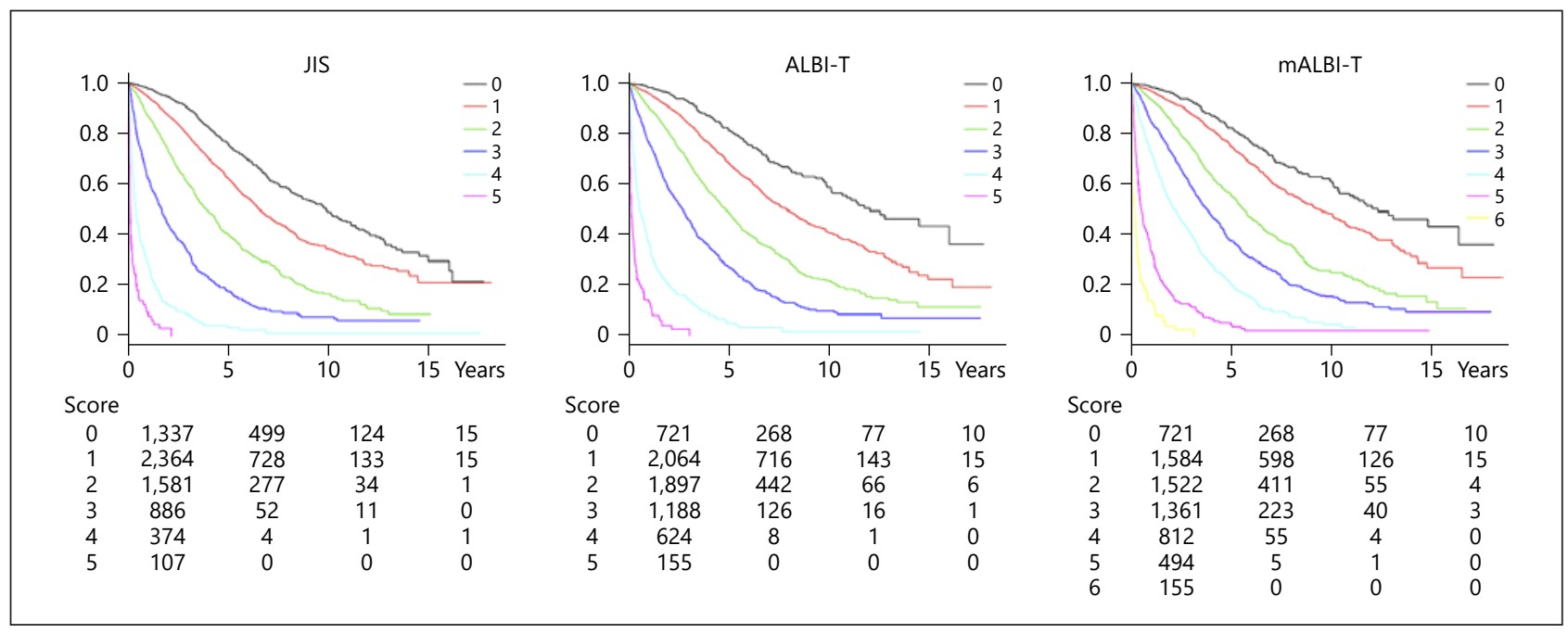

Fig. 2. JIS, ALBI-T, and mALBI-T scores in all patients. For all patients ( $n=6,649)$, the AIC and c-index values for JIS were 45,555.8 and 0.739, respectively, for ALBI-T were 45,467.7 and 0.744, respectively, and for mALBI-T were 45,327.1 and 0.755, respectively. mALBI-T was superior in both stratification and prognostic predictive ability, followed in order by ALBI-T and JIS for both. Values on the $y$ axes represent estimation of cumulative survival probability.

Table 4. Median survival time, AIC, and c-index for each scoring system

\begin{tabular}{llcc}
\hline & \multicolumn{2}{l}{ Median survival time, years $(95 \% \mathrm{CI})$} \\
\cline { 2 - 4 } & JIS & ALBI-T & mALBI-T \\
\hline Score 0 & $9.85(8.70-10.88)$ & $12.05(10.16-\mathrm{NA})$ & $12.05(10.16-\mathrm{NA})$ \\
Score 1 & $6.53(6.21-6.96)$ & $7.89(7.21-8.51)$ & $9.26(8.33-10.24)$ \\
Score 2 & $3.94(3.56-4.32)$ & $4.85(4.53-5.18)$ & $5.50(5.16-5.89)$ \\
Score 3 & $1.66(1.46-1.88)$ & $2.72(2.43-2.93)$ & $3.70(3.39-4.01)$ \\
Score 4 & $0.37(0.31-0.46)$ & $0.58(0.49-0.71)$ & $2.11(1.86-2.43)$ \\
Score 5 & $0.09(0.08-0.13)$ & $0.14(0.10-0.21)$ & $0.48(0.40-0.58)$ \\
Score 6 & none & none & $0.14(0.10-0.21)$ \\
\hline AIC & $45,555.8$ & $45,467.7$ & $45,327.1$ \\
c-index & 0.739 & 0.744 & 0.755 \\
\hline
\end{tabular}

JIS, Japan Integrated Staging; ALBI-T, albumin-bilirubin grade-TNM; mALBI-T, modified ALBI-T; AIC, Akaike's information criterion.

Holm's method) (data not shown). As a result, ALBI-T scoring (grade 1-4) could be separated in a more detailed manner (mALBI-T grade 1-5) by division of ALBI grade 2 into 2 subgrades. The AIC and c-index values for JIS were 45,555.8 and 0.739, respectively, while those for ALBI-T were 45,467.7 and 0.744, respectively, and for mALBI-T were 45,327.1 and 0.755, respectively. The correlation of median survival time with mALBI-T score was good as compared to each corresponding JIS score (Fig. 2) (Table 4). The result of reevaluation of hepatic function using mALBI grade was shown in Figure 3. Thus, mALBI grade has a better assessment ability than ALBI grade and Child-Pugh classification, and mALBI-T showed the best stratification ability and prognostic predictive value, followed in order by ALBI-T and JIS for both values, as a result (Table 4). 


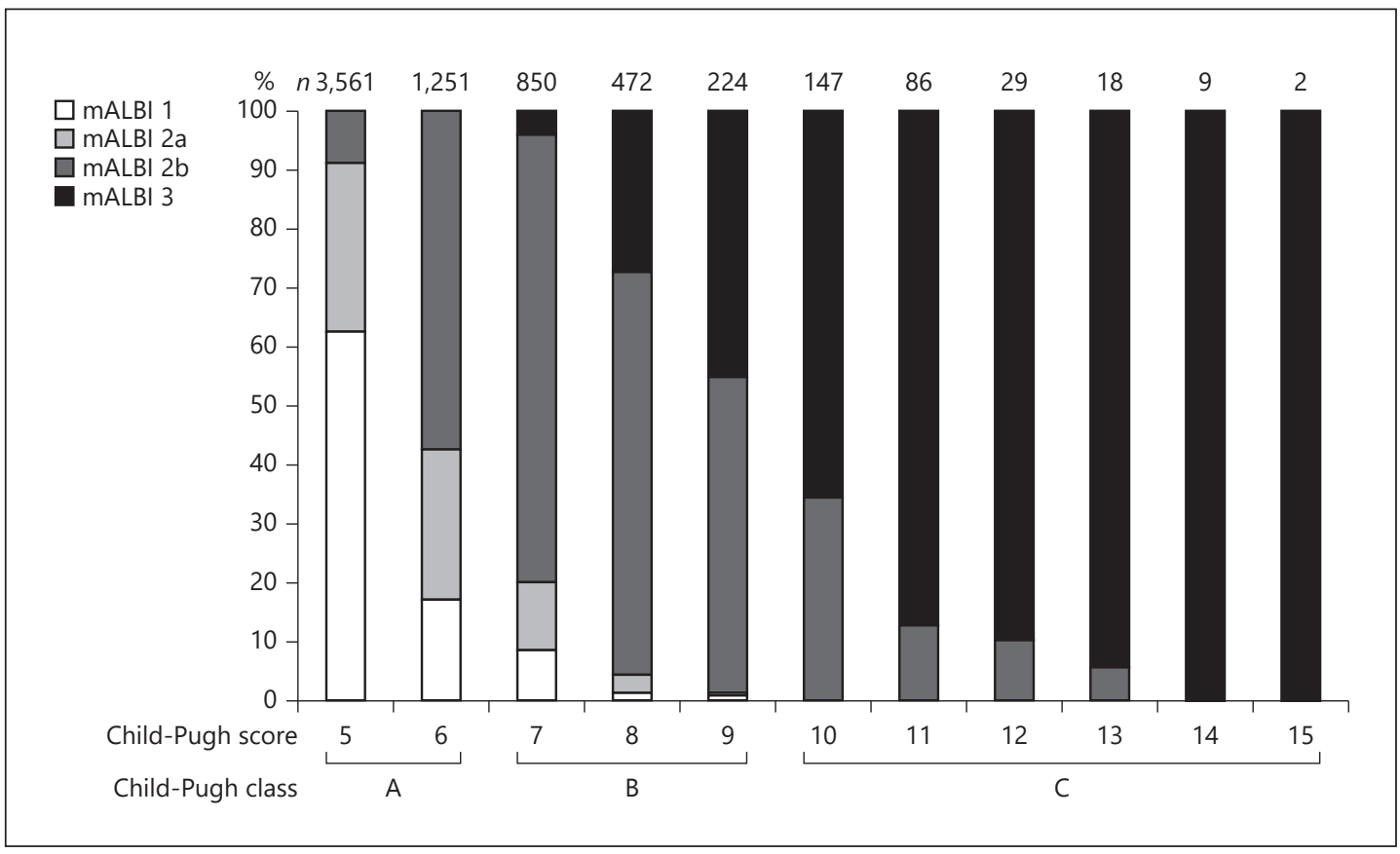

Fig. 3. Reevaluation of reserve function using modified albumin-bilirubin (mALBI) grade.

\section{Discussion}

It is well known that the prognosis of HCC patients is dependent on hepatic reserve function and tumor burden [26, 27]. Recently established treatment strategies for HCC [23, $24,28]$, as well as progression of therapeutic and diagnostic modalities (e.g., resection, low invasive RFA [6-8], CEUS [20, 21], EOB-MRI [18, 19]) have contributed to improve the prognosis of affected patients. Moreover, improved antiviral therapies (interferon [29], direct acting antivirals [2, 3], nucleotide analogues [4, 5]) have been shown to be helpful for increasing hepatic reserve function in cases of viral-related HCC. In addition, there has been a rapid increase in the proportion of cases negative for HBV and HCV, so-called NBNC-HCC, with the rate of incidence increasing steadily from $8.7 \%$ in 2000 to $14.9 \%$ in 2007 in Japan [30], with a more recent report noting that the rate is now greater than $20 \%$ [31]. As a result, the frequency of patients with better Child-Pugh class has increased, which is considered to be a recent trend in HCC cases treated in Japan [32]. Thus, in this era, the necessity of a suitable assessment tool for hepatic function has been increasing, with ALBI grade based on a statistical method proposed for helping to meet the present clinical need [10]. In a recent study, the predictive ability of ALBI-T, determined using ALBI grade and TNM stage, for the prognosis of HCC patients was shown to be better than that of JIS, which is determined based on Child-Pugh class and TNM stage [11,33]. ALBI grade is thought to be a suitable assessment tool for hepatic function.

On the other hand, ALBI grade has also a weak point as well as Child-Pugh classification. An issue with Child-Pugh classification is that hepatic function of the middle group (ChildPugh B) has a wide range of scores [7-9]. However, division of class B is difficult because the factors used for this classification are not defined statistically and have variability. Although ALBI grade 2 also features a wide range similar to Child-Pugh class B, an advantage is that ALBI grade is defined by serial ALBI scores, which are calculated statistically using only serum 
albumin and bilirubin levels. In previous reports, a cut-off value for ALBI score $(-2.270)$ for dividing into subgrades $2 \mathrm{a}$ and $2 \mathrm{~b}$ [15] was proposed based on indocyanine green retention rate after 15 min (ICG-R15) (cut-off value 30\% or less), which is an indicator for use of a subsegmentectomy as a minimum anatomical resection in the Makuuchi criteria [34, 35]. In the present validation study, mALBI-T showed both a more detailed stratification ability and better predictive value for prognosis of HCC patients as compared to those shown by ALBI-T and JIS.

The present study has some limitations, including its retrospective nature. Furthermore, the participating institutions are high-volume treatment centers; thus, the number of patients not indicated for treatment for HCC was small at each. Findings from a prospective study are needed before definite conclusions can be made.

In summary, although mALBI grade is made of only 2 factors (albumin and total bilirubin), it showed an ability for more detailed stratification of hepatic reserve function, which is valuable for the recent trend seen in HCC patients. We consider that mALBI-T may provide more accurate prediction of prognosis of affected patients as compared to existing total staging scoring systems.

\section{Disclosure Statement}

The authors declare no conflicts of interest.

\section{References}

1 GLOBOCAN: 2012. Estimated breast cancer incidence, mortality and prevalence worldwide in 2012. http:// globocan.iarc.fr/Pages/fact_sheets_cancer.aspx (accessed January 17, 2018).

2 Omata M, Nishiguchi S, Ueno Y, Mochizuki H, Izumi N, Ikeda F, Toyoda H, et al: Sofosbuvir plus ribavirin in Japanese patients with chronic genotype 2 HCV infection: an open-label, phase 3 trial. J Viral Hepat 2014;21: $762-768$.

3 Ogawa E, Furusyo N, Nomura H, Dohmen K, Higashi N, Takahashi K, Kawano A, et al: NS5A resistance-associated variants undermine the effectiveness of ledipasvir and sofosbuvir for cirrhotic patients infected with HCV genotype 1b. J Gastroenterol 2017;52:845-854.

4 Hiraoka A, Michitaka K, Kumagi T, Kurose K, Uehara T, Hirooka M, Yamashita Y, et al: Efficacy of lamivudine therapy for decompensated liver cirrhosis due to hepatitis B virus with or without hepatocellular carcinoma. Oncol Rep 2005;13:1159-1163.

5 Hosaka T, Suzuki F, Kobayashi M, Seko Y, Kawamura Y, Sezaki H, Akuta N, et al: Long-term entecavir treatment reduces hepatocellular carcinoma incidence in patients with hepatitis B virus infection. Hepatology 2013;58: 98-107.

6 Tateishi R, Shiina S, Teratani T, Obi S, Sato S, Koike Y, Fujishima T, et al: Percutaneous radiofrequency ablation for hepatocellular carcinoma. An analysis of 1,000 cases. Cancer 2005;103:1201-1209.

7 Hiraoka A, Horiike N, Yamashita Y, Koizumi Y, Doi K, Yamamoto Y, Hasebe A, et al: Efficacy of radiofrequency ablation therapy compared to surgical resection in 164 patients in Japan with single hepatocellular carcinoma smaller than $3 \mathrm{~cm}$, along with report of complications. Hepatogastroenterology 2008;55:2171-2174.

8 Shiina S, Tateishi R, Arano T, Uchino K, Enooku K, Nakagawa H, Asaoka Y, et al: Radiofrequency ablation for hepatocellular carcinoma: 10-year outcome and prognostic factors. Am J Gastroenterol 2012;107:569-577; quiz 578.

9 Pugh RN, Murray-Lyon IM, Dawson JL, Pietroni MC, Williams R: Transection of the oesophagus for bleeding oesophageal varices. Br J Surg 1973;60:646-649.

10 Johnson PJ, Berhane S, Kagebayashi C, Satomura S, Teng M, Reeves HL, O’Beirne J, et al: Assessment of liver function in patients with hepatocellular carcinoma: a new evidence-based approach-the ALBI grade. J Clin Oncol 2015;33:550-558.

11 Hiraoka A, Kumada T, Michitaka K, Toyoda H, Tada T, Ueki H, Kaneto M, et al: Usefulness of albumin-bilirubin grade for evaluation of prognosis of 2,584 Japanese patients with hepatocellular carcinoma. J Gastroenterol Hepatol 2016;31:1031-1036.

12 Shao YY, Liu TH, Lee YH, Hsu CH, Cheng AL: Modified CLIP with objective liver reserve assessment retains prognosis prediction for patients with advanced hepatocellular carcinoma. J Gastroenterol Hepatol 2016;31: 1336-1341. 
13 Chan AW, Kumada T, Toyoda H, Tada T, Chong CC, Mo FK, Yeo W, et al: Integration of albumin-bilirubin (ALBI) score into Barcelona Clinic Liver Cancer (BCLC) system for hepatocellular carcinoma. J Gastroenterol Hepatol 2016;31:1300-1306.

14 Hiraoka A, Kumada T, Kudo M, Hirooka M, Tsuji K, Itobayashi E, Kariyama K, et al: Albumin-Bilirubin (ALBI) Grade as part of the Evidence-Based Clinical Practice Guideline for HCC of the Japan Society of Hepatology: a comparison with the Liver Damage and Child-Pugh classifications. Liver Cancer 2017;6:204-215.

15 Hiraoka A, Michitaka K, Kumada T, Izumi N, Kadoya M, Kokudo N, Kubo S, et al: Validation and potential of albumin-bilirubin grade and prognostication in a nationwide survey of 46,681 hepatocellular carcinoma patients in Japan: the need for a more detailed evaluation of hepatic function. Liver Cancer 2017;6:325-336.

16 Hiraoka A, Michitaka K, Kumada T, Kudo M: ALBI score as a novel tool in staging and treatment planning for hepatocellular carcinoma: advantage of ALBI Grade for Universal Assessment of Hepatic Function. Liver Cancer 2017;6:377-379.

17 Bruix J, Sherman M: Management of hepatocellular carcinoma. Hepatology 2005;42:1208-1236.

18 Di Martino M, Marin D, Guerrisi A, Baski M, Galati F, Rossi M, Brozzetti S, et al: Intraindividual comparison of gadoxetate disodium-enhanced MR imaging and 64-section multidetector CT in the Detection of hepatocellular carcinoma in patients with cirrhosis. Radiology 2010;256:806-816.

19 Sano K, Ichikawa T, Motosugi U, Sou H, Muhi AM, Matsuda M, Nakano M, et al: Imaging study of early hepatocellular carcinoma: usefulness of gadoxetic acid-enhanced MR imaging. Radiology 2011;261:834-844.

20 Hiraoka A, Ichiryu M, Tazuya N, Ochi H, Tanabe A, Nakahara H, Hidaka S, et al: Clinical translation in the treatment of hepatocellular carcinoma following the introduction of contrast-enhanced ultrasonography with Sonazoid. Oncol Lett 2010;1:57-61.

21 Hiraoka A, Hiasa Y, Onji M, Michitaka K: New contrast enhanced ultrasonography agent: impact of Sonazoid on radiofrequency ablation. J Gastroenterol Hepatol 2011;26:616-618.

22 Liver Cancer Study Group of Japan: The General Rules for the Clinical and Pathological Study of Primary Liver Cancer, ed 6. Tokyo, Kanehara, 2015, p 26.

23 Makuuchi M, Kokudo N, Arii S, Futagawa S, Kaneko S, Kawasaki S, Matsuyama Y, et al: Development of evidencebased clinical guidelines for the diagnosis and treatment of hepatocellular carcinoma in Japan. Hepatol Res 2008;38:37-51.

24 Kokudo N, Hasegawa K, Akahane M, Igaki H, Izumi N, Ichida T, Uemoto S, et al: Evidence-based Clinical Practice Guidelines for Hepatocellular Carcinoma: The Japan Society of Hepatology 2013 update (3rd JSH-HCC Guidelines). Hepatol Res 2015;45.

25 Kanda Y: Investigation of the freely available easy-to-use software "EZR" for medical statistics. Bone Marrow Transplant 2013;48:452-458.

26 Kudo M, Chung H, Haji S, Osaki Y, Oka H, Seki T, Kasugai H, et al: Validation of a new prognostic staging system for hepatocellular carcinoma: the JIS score compared with the CLIP score. Hepatology 2004;40:1396-1405.

27 Kudo M, Chung H, Osaki Y: Prognostic staging system for hepatocellular carcinoma (CLIP score): its value and limitations, and a proposal for a new staging system, the Japan Integrated Staging Score (JIS score). J Gastroenterol 2003;38:207-215.

28 Omata M, Cheng AL, Kokudo N, Kudo M, Lee JM, Jia J, Tateishi R, et al: Asia-Pacific clinical practice guidelines on the management of hepatocellular carcinoma: a 2017 update. Hepatol Int 2017;11:317-370.

29 Asahina Y, Tsuchiya K, Tamaki N, Hirayama I, Tanaka T, Sato M, Yasui Y, et al: Effect of aging on risk for hepatocellular carcinoma in chronic hepatitis C virus infection. Hepatology 2010;52:518-527.

30 Ikai I, Arii S, Okazaki M, Okita K, Omata M, Kojiro M, Takayasu K, et al: Report of the 17th Nationwide Followup Survey of Primary Liver Cancer in Japan. Hepatol Res 2007;37:676-691.

31 Hiraoka A, Ochi M, Matsuda R, Aibiki T, Okudaira T, Kawamura T, Yamago H, et al: Ultrasonography screening for hepatocellular carcinoma in Japanese patients with diabetes mellitus. J Diabetes 2016;8:640-646.

32 Hiraoka A, Hidaka S, Shimizu Y, Utsunomiya H, Imai Y, Tatsukawa H, Tazuya N, et al: Recent trends of Japanese hepatocellular carcinoma due to HCV in aging society. Hepatogastroenterology 2012;59:1893-1895.

33 Hiraoka A, Kumada T, Hirooka M, Tsuji K, Itobayashi E, Kariyama K, Ishikawa T, et al: A better method for assessment of hepatic function in hepatocellular carcinoma patients treated with radiofrequency ablation: usefulness of albumin-bilirubin grade. Hepatol Res 2018;48:E61-E67.

34 Miyagawa S, Makuuchi M, Kawasaki S, Kakazu T: Criteria for safe hepatic resection. Am J Surg 1995;169:589594.

35 Seyama Y, Kokudo N: Assessment of liver function for safe hepatic resection. Hepatol Res 2009;39:107-116. 